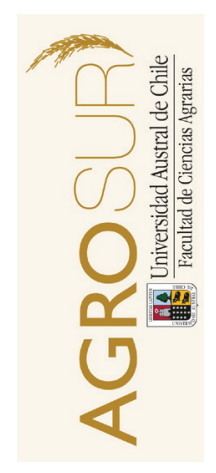

\title{
Contenido y estabilidad de clorofilas y carotenoides en el zumo de lechuga (Lactuca sativa L.)
}

\author{
Content and stability of chlorophyll and carotenoids \\ in lettuce juice (Lactuca sativa L.)
}

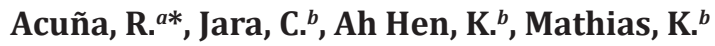 \\ ${ }^{a}$ Instituto de Producción y Sanidad Vegetal, Facultad de Ciencias Agrarias, Universidad Austral de Chile. \\ Casilla 567, Valdivia, Chile.
}

${ }^{b}$ Instituto de Ciencia y Tecnología de los Alimentos Facultad de Ciencias Agrarias, Universidad Austral de Chile. Casilla 567, Valdivia, Chile.

\begin{tabular}{l} 
A R T I C L E I N F O \\
\hline Article history: \\
Received 15.01.2018 \\
Accepted 18.04.2018 \\
\hline Keywords: \\
Lettuce \\
Juice \\
Functional compounds \\
Chlorophylls \\
Carotenoids \\
Spectrophotometry \\
Preservative \\
\hline Original Research Article, \\
Food Processing and Quality \\
\hline *Corresponding author: \\
Rodrigo Acuña \\
E-mail address: \\
rodrigoacuna@uach.cl
\end{tabular}

\begin{abstract}
A B S T R A C T
Degradation dynamics was studied in a new antioxidant format to use natural juice of lettuce (Lactuca sativa $\mathrm{L}$., $\mathrm{Cv}$. Justine) in human diets as a functional food. Chlorophylls $a, b$ and carotenoids content and stability were quantified and characterized under $\mathrm{pH}$-medium and temperature conservation techniques for ten days. A 3x3 factorial ANOVA arrangement design was used. The first and second factors were the juice storage temperature $\left(5,20\right.$ and $35^{\circ} \mathrm{C}$ ), and the liquid pH-medium (without additives, 4,5 and 7,5), respectively. Significant minimum differences were achieved by means of Tukey's multiple comparison test $(\alpha=0.05)$.

Significant differences were found on pigment concentrations between $\mathrm{pH}$ factor versus temperature. The storage temperature $\mathrm{x}$ liquid $\mathrm{pH}$-medium interaction was signficant and showed that chlorophylls $a$ and $b$ and carotenoids maintained greater stability in alkaline solutions and temperatures close to $20^{\circ} \mathrm{C}$. The most important conservation factor was $\mathrm{pH}$. The results allowed to conclude that there is wide variation between pigment types, highlighting chlorophyll $b$ that had a more desirable behavior at higher temperatures. In general, there were evident decreases in pigment concentration from five days of storage, suggesting a potential use of lettuce juice as a functional ingredient in human diets.
\end{abstract}

\section{RESUMEN}

Se estudió la dinámica degradativa de nuevos formatos de consumo de pigmentos antioxidantes para beneficio de la salud humana. Se cuantificó y caracterizó la estabilidad de clorofilas $a$, $b$ y carotenoides en zumo de Lechuga (Lactuca sativa L.) Cv. Justine durante diez días. Extractos de jugos se conservaron en base a diferentes pH y temperaturas. Se utilizó un diseño experimental ANDEVA con arreglo factorial de 3x3. El primer factor correspondió a la temperatura de almacenamiento del zumo (5, 20 y $35^{\circ} \mathrm{C}$ ), y el segundo al pH del medio (sin aditivos, 4,5 y 7,5). Diferencias mínimas significativas se midieron utilizando el test de Tukey $(\alpha=0,05)$.

Se encontraron diferencias significativas sobre concentraciones de los pigmentos entre el factor $\mathrm{pH}$ frente al efecto de la temperatura. Los resultados de la interacción de los factores mostraron que las clorofilas $a$ y $b$ y carotenoides, mantuvieron mayor estabilidad en soluciones alcalinas y en condiciones de temperaturas cercanas a los $5{ }^{\circ} \mathrm{C}$, identificándose además variabilidad asociada a las características propias de la molécula. El factor más importante para conservación fue el pH. Los resultados permitieron concluir que existe variación en la degradación de acuerdo al tipo de pigmentos, destacando la clorofila $b$ que tuvo un comportamiento más deseable a temperaturas mayores. En general se evidenciaron disminuciones notorias en la concentración de pigmentos en el zumo desde los tres a cuatro primeros días de almacenaje. Los resultados permiten suponer los tiempos de conservación del zumo y también los momentos de inflexión de la degradación para mejorar el resultado cuantitativo final.

Palabras clave: Lechuga, jugo, componentes funcionales, clorofilas, carotenoides, espectrofotometría, preservantes.

\section{INTRODUCCIÓN}

Las hortalizas de hoja verde contienen varios tipos de pigmentos dentro de los que destacan las clorofi- las (Chl) del tipo a y $b$, los carotenoides ( $\alpha$ y $\beta$ ) (Sangeetha y Baskaran, 2010; Liu et al., 2007) y también otros fitoquímicos naturales como fenoles y flavonoides totales, vitamina $\mathrm{C}$ y licopenos (Dewanto et al., 
2002) que al consumirlos, previenen dolencias coronarias (Engelhard et al., 2006) o enfermedades como el cáncer (Bertram y Bortkiewicz, 1995; Prior y Cao, 2000; Liu, 2004). Su efecto en el consumo se asocia a la capacidad de evitar el estrés oxidativo ante la presencia de radicales libres (RL) (Burns et al., 2003; Liu, 2004; Carvalho et al., 2006; Khoo et al., 2011; Clark y Lee, 2016), por lo que su disponibilidad condiciona la calidad de los productos vegetales (Koca et al., 2007), lo que justifica su inclusión en las dietas como estrategia de control preventivo de las dolencias mencionadas (Winograd, 2006; MINSAL, 2016). El resultado de acción de estos pigmentos dependen del potencial reductor de diferentes tipos de moléculas presentes en células, tejidos y órganos de quien los consume, por lo que se hace necesario conocer y diferenciar el potencial oxidante de las mismas en base a sus RL.

Los RL son moléculas reactivas e inestables energizadas con electrones no apareados y se encuentran entre otros, en alimentos que contienen ácidos grasos insaturados. Ejemplo de RL son los derivados del oxígeno que incluyen al superóxido $\left(\mathrm{O}_{2}^{-}\right)$, hidroxilo $\left(\mathrm{OH}^{-}\right)$, hidroperoxilo (HOO), peroxilo (ROO) y alcoxilo (RO). También los hay en base al óxido nítrico (NO-) y al anión peroxinitrato $\left(\mathrm{ONOO}^{-}\right)$, entre tantos más (Prior y Cao, 2000). Los RL reaccionan rápidamente con los compuestos circundantes dónde reducen electrones debido a su mayor potencial electroquímico. Para el caso de las células, una vez que se inicia el proceso de peroxidación, se produce una desestabilización en cadena conducente a una desintegración de membranas que afecta a las proteínas y al ADN, resultando en la alteración estructural y funcional de esta unidad básica (Halliwell et al., 1995).

Se sabe que la temperatura de almacenamiento es el factor más crítico para mantener la calidad en el proceso de productos vegetales (Hall et al., 2013). Valores entre 0 y $10^{\circ} \mathrm{C}$ ayudan a reducir la proliferación microbiana, respiración y degradación de compuestos bioactivos, sin embargo, el efecto final depende del tipo de material vegetal en estudio (Santos et al., 2012). Los protocolos usualmente conllevan además variaciones de $\mathrm{pH}$, a las que se suman a las transformaciones o degradaciones naturales que se producen durante la senescencia de la planta como también las estacionales de acuerdo a la época de producción (Kufner, 1980; Gunawan y Barringer, 2000).

Los pigmentos, particularmente las Chl $a$ y $b$ se encuentran en una relación aproximada de 3:1 en plantas superiores, y son responsables del color verde característico en las hojas. Entre las Chl difieren en que la $a$ poseen un grupo metilo en lugar de un residuo de aldehído (-CHO) como en la $b$ en la posición 3 del carbono, lo que promueve en esta última resistencia o estabilidad térmica mayor (Schoefs, 2002). Dentro este grupo, se pueden diferenciar a las $a$ y $a^{\prime}$ que son altamente intercambiables a temperatura ambiente y mucho más aún a temperaturas de 95 a $100{ }^{\circ} \mathrm{C}$ (Gross, 1991). En estos procesos, la molécula de clorofila pierde su átomo central de Mg transformándose en feofitinas $a$ y $b$ (Pennington et al., 1964), que otorgan las coloración oliva posterior a los tratamientos térmicos.

La clorofila y los carotenoides son altamente reactivas al oxígeno debido a la presencia de una cadena larga de dobles enlaces de carbono conjugados (Chen y Chen, 1993), por lo que otra estrategia para conservar el pigmento es intervenir en la cadena metabólica o las enzimas que actúan sobre este blanco. Se busca la desnaturalización de las enzimas asociadas (peroxidasas) por medio de la pasteurización. Estos métodos también ayudan a eliminar gases, sustancias sápidas no deseables y producir ablandamiento de tejidos, pero colateralmente induce la degradación de la clorofila (Hernández y Gallego, 1999; Tijskens et al., 2001; Updike y Schwartz, 2003). Otro factor a considerar en la estabilidad de la molécula es la luz. Maunders y Brown (1983), determinaron la estabilidad de la clorofila en hojas separadas de la planta y mantenidas en la oscuridad vs otras bajo luz natural, observando en las primeras una descomposición significativamente menor durante un periodo de 14 días, siendo siempre la Chl $a$ más sensible a la degradación. La explicación más probable es que la luz tiene un papel fotoquímico directo en la degradación de clorofila en hojas senescentes, y que dicha fotodegradación, en lugar de reacción metabólica, puede ser la principal responsable de la pérdida estacional de clorofila durante la senescencia foliar .

En general se estima que para todos los pigmentos, la desnaturalización o conversión de ellos se produce por temperaturas umbrales de 50 a $60{ }^{\circ} \mathrm{C}$ variando su tasa de deterioro en relación a la duración del tratamiento, especie vegetal y pH del medio (Gross, 1991).

Los carotenos son insolubles en agua, lipofílicos, y se consideran como Provitaminas A (Shi y Le Maguer, 2000). También son altamente susceptibles a la degradación durante el procesamiento térmico (Schwartz y Von Elbe, 1983), lo que altera las propiedades que otorgan atributos de calidad visual y organoléptica a estos productos (Hayakawa y Timbers, 1977; Xue y Yang, 2009), sobre todo asociado a coloraciones amarillas, naranjas y rojas (Arias et al., 2000). El contenido de carotenoides también cambia con la intensidad de la luz, manejos de precosecha, estado madurez del producto y la presencia de oxígeno al momento del proceso del producto (Meléndez-Martínez et al., 2007).

Por otra parte, la mayor cantidad de los alimentos podrían conservarse en buenas condiciones microbiológicas cuando el medio tiene un $\mathrm{pH} 4,0$, de modo que se han desarrollado una serie de métodos que persiguen controlar el $\mathrm{pH}$ mediante la producción endógena de ácido o por adición exógena de algún ácido 
orgánico como el acético, el cítrico e incluso el láctico (FAO, 1993). Sin embargo, se sabe que el pH inicial del material vegetal y los cambios del $\mathrm{pH}$ en el medio durante el proceso y almacenamiento son factores determinantes en la degradación de la clorofila, por lo que se busca ajustar el pH con agentes alcalinizantes utilizando sales orgánicas e inorgánicas (Andrés-Bello et al., 2013). En espinacas se ha observado una mejora en la estabilidad de la clorofila con el uso de carbonato de magnesio durante el almacenamiento mantiéndose las condiciones de calidad cuando el pH es elevado, mientras que por el contrario, la clorofila se vuelve verde oliva en medios ácidos (Greve et al., 1994).

Los contenidos de pigmentos en lechugas varían de acuerdo a los cultivares, estado de madurez e incluso los métodos analíticos aplicados en su determinación (Izaki et al., 1986). Para el caso de los carotenos, estos autores reportaron variaciones de hasta un 1400\% entre variedades, detallando valores medios de 11,95 y $3,5 \mu \mathrm{g} \beta$-caroteno $\mathrm{g} \mathrm{MS}^{-1}$ en hojas maduras y nuevas, respectivamente, lo que constituye una diferencia del $340 \%$ superiores en hojas maduras respecto a las hojas jóvenes. Las clorofilas $a$ y $b$ en tanto tienen una misma tendencia de variación de acuerdo a los cultivares seleccionados variando de 5,3 a 74,9 y 1,5 a $16 \mathrm{mg} 100 \mathrm{~g}$ $\mathrm{MS}^{-1}$, respectivamente. Lo anterior cobra importancia, pues las valorizaciones económicas y de calidad se hacen en términos de colorimetría (Koca et al., 2007) y pérdida degradación porcentual de la molécula, y no sobre el contenido absoluto de Chl durante el almacenamiento (Gross, 1991).

Otra fuente de variación sobre los contenidos absolutos de Chl en lechugas estan dados por la estación de cultivo, maximizándose su contenido en verano, pudiendo ser en estas condiciones cuatro veces superior en concentración de la $\mathrm{Chl} a$ con respecto a la $b$, mientras que en el invierno la relación puede incluso ser 1:1 (Kufner, 1980), por lo que para efectos industriales, se debería considerar con mayor fuerza utilizar el criterio de la cantidad bruta y su estabilidad en el tiempo como medida de calidad del producto, más allá de la colorimetría o variación porcentual por almacenamiento.

Pese a la toda información anterior, es muy poco frecuente hallar en la literatura protocolos o valores referenciales de ingesta de zumos o jugos de hojas de hortalizas que puedan ser considerados por su potencial nutracéutico, y que se incluyan para dar valor agregado a los alimentos de consumo cotidiano. Por esta razón se planteó como objetivo evaluar el contenido y estabilidad de los pigmentos fotosintéticos (clorofilas $a, b$ y carotenoides) hallados en licuados provenientes de hojas de lechugas para consumo directo o complemento para mejorar el nivel de funcionalidad en otros alimentos. Se planteó que modificando las condiciones medioambientales de conservación en cuanto a pH y temperatura del zumo de lechuga, se pueden mantener sus compuestos bioactivos (pigmentos) por tiempos diferenciales.

\section{MATERIAL Y MÉTODOS}

La investigación se realizó en los laboratorios del Instituto de Ciencia y Tecnología de los Alimentos (ICYTAL) de la Facultad de Ciencias Agrarias de la Universidad Austral de Chile, Valdivia, durante un período de almacenamiento de diez días.

\section{Materiales y equipos}

Se utilizaron lechugas (Lactuca sativa L.) var. Capitata (tipo mantecosas) cv. Justine, las cuales fueron recolectadas en madurez de consumo a inicios del verano desde parcelas experimentales de la Estación Experimental Agropecuaria Austral - Fundo Santa Rosa, ubicada en el sector Cabo Blanco, perteneciente a la misma Universidad. Luego de realizar los protocolos de saneamiento, separación y procesado de higiene de las hojas para garantizar la inocuidad del producto (Hormazabal, 1999), y extracción del zumo (FAO, 1993), se determinaron las variables del experimento: contenido de pigmentos $\left(\mu \mathrm{L} \mathrm{L}^{-1}\right)$ y tasa de degradación diaria (\% deg).

La extracción de jugo se hizo con ayuda de un extractor de jugos (Sindelen Mod EX350, RPC) que separó la pulpa y el jugo del producto no agregando agua en el proceso. Posteriormente, se envasó el zumo en tubos Falcon de $20 \mathrm{~mL}$, y se pasteurizó a $65^{\circ} \mathrm{C} 25 \mathrm{~min}^{-1}$ para eliminar/inactivar la flora microbiana y enzimas que pudieran causar el deterioro del jugo. Luego, los tubos fueron introducidos en una tina con agua a $5^{\circ} \mathrm{C}$ hasta alcanzar la misma temperatura para hacer efectiva la pasteurización. Posteriormente, los tubos fueron llevados a condiciones de $-18^{\circ} \mathrm{C}$ (Mademsa, Mod. MFH x 2000, CL) para conservar las propiedades hasta el momento de iniciar los análisis.

La extracción de pigmentos para cada réplica se realizó incorporando el jugo a un vaso precipitado de $100 \mathrm{~mL}$ con $0,4 \mathrm{~g}$ de $\mathrm{Ca}_{2} \mathrm{CO}_{3}$ precipitado. Posteriormente, se sacaron $20 \mathrm{~mL}$ de la solución y en un vaso precipitado de $50 \mathrm{~mL}$, se diluyeron en $10 \mathrm{~mL}$ de acetona $85 \% \mathrm{v} / \mathrm{v}$. Enseguida se adicionaron $5 \mathrm{~mL}$ de éter de petróleo $0,1 \mathrm{~N}$ hasta que visualmente toda la pigmentación de la grasa y fibra soluble se hubiese separado. Finalmente, se incorporaron $50 \mathrm{~mL}$ de $\mathrm{H}_{2} \mathrm{O}$ destilada a la solución. El extracto fue filtrado a través de un disco de papel filtro (Whatman Grado 41, Ashless $150 \mathrm{~mm}$, Schleicher \& Schuell, Alemania) y el producto se incorporó a las cubetas espectrofotométricas. Las lecturas de concetración de pigmentos para clorofilas $a$ y $b, \mathrm{y}$ carotenoides se hicieron introduciendo estas cubetas en un espectrofotómetro (Ray Leigh, Mod. UV-1601, 
RPC) calibrado y en forma periódica cada $24 \mathrm{~h}$, expresando los resultados como $\mu \mathrm{g} \mathrm{mL}^{-1}$ de acuerdo a los patrones de densidad óptica de 660, 642 y $470 \mathrm{~nm}$ para las clorofilas $a, b$ y carotenoides, respectivamente (Lichtenthaler, 1987). Las concentraciones de clorofila en las muestras fueron calculadas de acuerdo a las siguientes ecuaciones (AOAC, 1980):

$$
\begin{gathered}
\operatorname{Chl}_{a}\left(\mu \mathrm{g} \mathrm{mL}^{-1}\right)=9,93 * \mathrm{~A}_{660}-0,777 * \mathrm{~A}_{642,5} \\
\mathrm{Chl}_{b}\left(\mu \mathrm{g} \mathrm{mL}{ }^{-1}\right)=17,6 * \mathrm{~A}_{642,2}-2,81 * \mathrm{~A}_{660}
\end{gathered}
$$

Mientras que para los carotenoides totales se utilizó la expresión (Lichtenthaler, 1987):

$\operatorname{Car}\left(\mu \mathrm{g} \mathrm{mL}^{-1}\right)=\frac{\left[1000 * \mathrm{~A}_{470}-1,82 * \mathrm{Chl}_{a}-\left(85,02 * \mathrm{Chl}_{b}\right)\right]}{198}$

La tasa de degradación de los pigmentos se obtuvo por medio de relaciones porcentuales entre los días estudiados, calculándose además el coeficiente de variación, en cada uno de los tratamientos a modo de comparar la varibilidad de la estabilidad.

Para inducir el cambio de pH en el jugo extraído, se utilizó ácido ascórbico y bicarbonato de sodio en una dosis de 2,5 g $100 \mathrm{~mL}$ de jugo de acuerdo a los regualdores ácido/base permitidos por el Reglamento Sanitario de los Alimentos del Ministerio de Salud de Chile (MINSAL, 1996). La temperatura para almacenamiento fue lograda con el uso de estufa (Memmert, Mod. 100-800, Alemania), siendo mantenidos los tubos en oscuridad y sacando una alícuota cada 24 horas para la determianción de las concentraciones de pigmentos.

\section{Diseño experimental}

Para el diseño experimental se utilizó un Análisis de Varianza (ANDEVA) con arreglo factorial de $3 \times 3$ $(\alpha=0,05)$, siendo el primer factor la temperatura de almacenamiento del jugo con subniveles de 5,20 y $35^{\circ} \mathrm{C}$; y el segundo, el conservante (ácido, básico, sin conservante o control), obteniendo nueve tratamientos los cuales contaron con tres réplicas (tubos) cada uno siendo esta la unidad experimental. Luego de comprobar los supuestos del modelo, se estudiaron las posibles interacciones entre los factores y en el caso de haber diferencias se realizó una segregación de a acuerdo a las diferencias mínimas con el Test de Tukey.

\section{RESULTADOS Y DISCUSIÓN}

Para todas las muestras se consideró un valor medio incial previo a las pasteurizaciones de $24,7 \mu \mathrm{g} \mathrm{L}^{-1}$ de clorofilas totales $\left(15,4\right.$ y $9,3 \mu \mathrm{g} \mathrm{L}^{-1}$ para Chl $a$ y $b$, respectivamente) y $15,1 \mu \mathrm{g} \mathrm{L}^{-1}$ de carotenoides.

\section{Efecto de la temperatura sobre los pigmentos: estabilidad y contenidos}

\section{Chl a}

Las dinámicas medias asociadas a regresiones logarítimicas significativas de degradación de la Chl $a$ en los distintos medios de conservación y para cada una de las temperaturas de almacenamiento, pueden ser vistas en la Figura 1 (A, B y C). Los datos arrojaron medias estadísticamente significativas para el factor temperatura con 5,$4 ; 3,5$ y $4,1 \mu \mathrm{g} \mathrm{L}^{-1}$, para $5,20,35^{\circ} \mathrm{C}$, respectivamente, para el primer día de almacenaje. Para el día cuatro, todos los tratamientos disminuyeron en torno a un $50 \%$, con valores medios significativos de 3,2; 2,3 y $2,7 \mu \mathrm{g} \mathrm{L}^{-1}$, en el mismo orden. El último día $\left(10^{\circ}\right)$, se obtuvieron los menores valores medios significativos entre si con degradaciones del 84,9; 94,3 y $91,3 \%$ con respecto al inicio del ensayo para las temperaturas de 5,20 y $30^{\circ} \mathrm{C}$, respectivamente (Cuadro 1 ). El promedio diario de Chl $a$ para los 10 días de almacenaje varió de un máximo de $3,3\left(5^{\circ} \mathrm{C}\right)$ a un mínimo de $2,1\left(20^{\circ} \mathrm{C}\right) \mu \mathrm{g} \mathrm{L}{ }^{-1}$, lo que significó un $55 \%$ más de pigmentos asociados al nivel de temperatura de $5^{\circ} \mathrm{C}$, siendo el valor para $35^{\circ} \mathrm{C}$ un punto intermedio con $2,8 \mu \mathrm{g}$ $\mathrm{L}^{-1}$ de Chl $a$. En general, aunque resultaron significativos todos los datos de comparaciones, el efecto de la temperatura en la degradación o estabilidad de la Chl $a$ fue mínimo, ya que la como promedio en el perído sólo fue responsable del 7,6\% de la variabilidad de acuerdo a la suma de cuadrados obtenidos en el periodo en el uso del ANDEVA.

\section{Chl b}

Las curvas logarítmicas significativas de degradación de la Chl $b$ promedio de los distintos medios de conservación y para cada una de las temperaturas de almacenamiento, pueden ser vistas en la Figura 1 (D, E y F), donde todos los valores fueron significantes entre los diferentes niveles térmicos de almacenamiento para cada uno de los días. El promedio observado de todos los conservantes al primer día de almacenamiento de Chl $b$ para $5{ }^{\circ} \mathrm{C}$ fue 6,9 , mientras que para $20^{\circ} \mathrm{C}$ se alcanzó $4,8 \mu \mathrm{g} \mathrm{L}^{-1}$, no pudiendo obtener el dato para los $35^{\circ} \mathrm{C}$ por pérdida de uno de los tratamientos ácido/ base. Para el cuarto día, los valores medios fueron 5,1; 3,1 y $3,6 \mu \mathrm{g} \mathrm{L}^{-1}$ para 5,20 y $35^{\circ} \mathrm{C}$, respectivamente. Al final del periodo de estudio, los valores también significantes, fueron $2,7 \mathrm{a} ; 1,1 \mathrm{~b}$ y $3,8 \mathrm{c}$ en el mismo orden. Del mismo modo que para la Chl $a$, el nivel de degradación medio del periodo asociado a este factor es bajo alcanzando sólo un 7,7\%, observándose que los máximos cambios corresponden en definitiva al factor de medio de almacenamiento. Hayakawa y Timbers (1977), describieron que un calentamiento en alimentos provoca 
pérdidas en la calidad organoléptica de los productos, y en este ensayo se observó esa tendencia no lineal. Para ambas clorofilas, resulta interesante observar que la máxima degradación se produjo con una temperatura ambiente de almacenamiento $\left(20^{\circ} \mathrm{C}\right)$, lo que probablemnte se debió al catabolismo por parte de las clorofilasas y peroxidadas excluyendo el efecto de microbios debido al tratamiento de pasteurización (Santos et al., 2012). Las enzimas se habrían inactivado a bajas temperaturas $\left(5^{\circ} \mathrm{C}\right)$, comenzando su desnaturalziación cuando estaban a $35^{\circ} \mathrm{C}$ (Fennena et al., 2010). También llama la atención el nivel de degradación medio de las clorofilas entre todas las temperaturas. Para el día uno, la degradación promedio entre los nieveles térmicos alcanzó un 71,6 vs. un 46,8\% para las Chl $a$ y $b$, respectivamente, mientras que en el último día los valores medios de degradación llegaron a 90,1 y 76,4\% en el mismo orden (Cuadro 1), lo cual parece indicar que la resistencia de la $\mathrm{Chl} b$ es mayor a las variaciones térmicas que la $\mathrm{Chl} a$, lo que es concordante con la termorresistencia de la molécula reportada por Canjura et al. (1991) en hojas de espinaca, y que se asociaría a la presencia en la molécula de un grupo aldheído (-CHO) que se sabe promueve una mayor resistencia térmica (Schoefs, 2002). Por otra parte, las proporciones de clorofilas mencionadas en la literatura $(3: 1-\mathrm{Chl} a$ :Chl $b$; Gross, 1991) no fueron observadas en este ensayo, lo que probablemente se debió al método de extracción del material. De acuerdo a lo anterior, se esperarían valores mínimos de $30 \mu \mathrm{g} \mathrm{L}^{-1}$ para la Chl $a$.

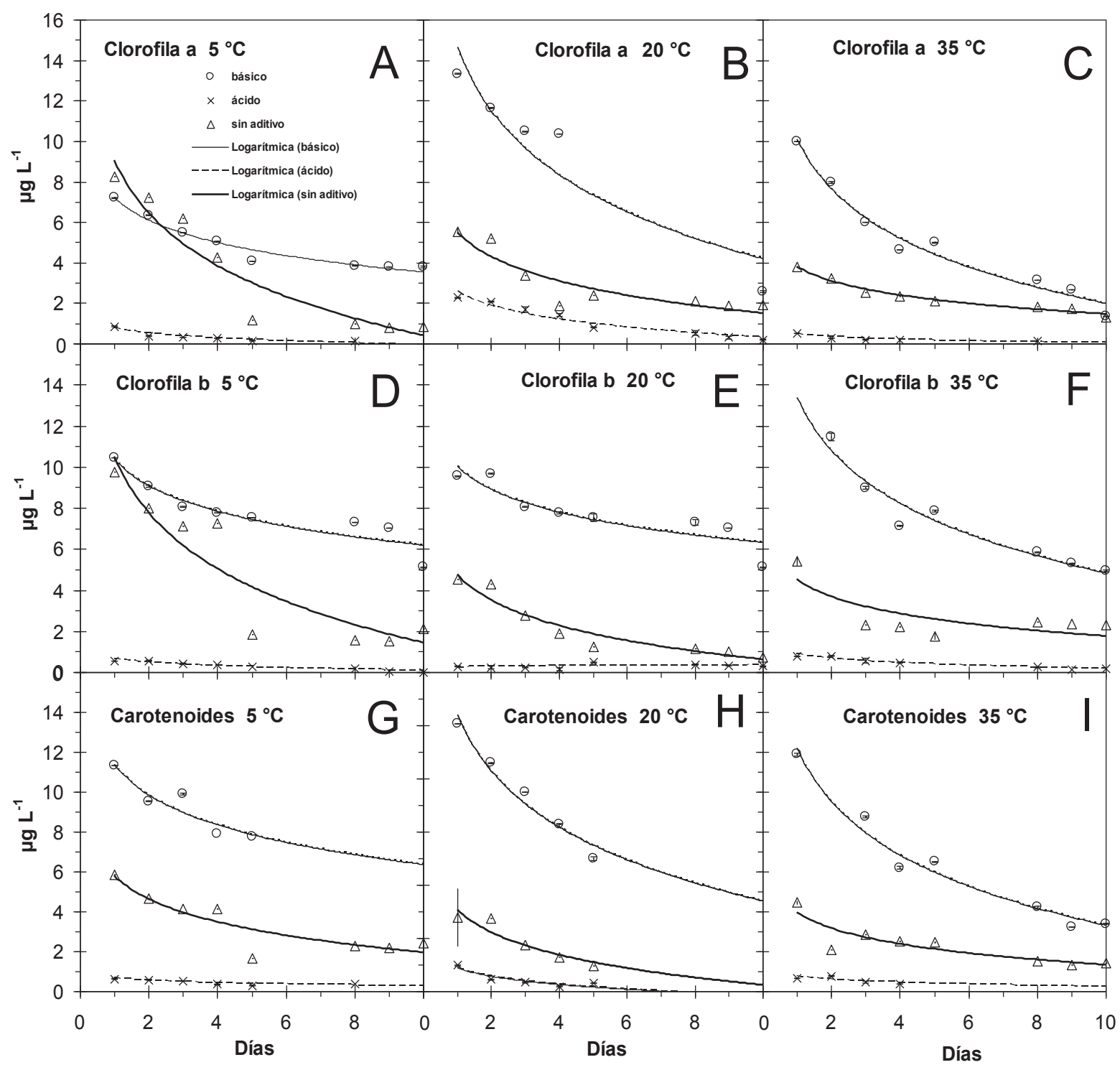

Figura 1. Pigmentos vs. temperatura con niveles del conservante, en función de los días de almacenamiento.

Figure 1. Pigments vs. temperature with preservative levels as a function of the days of storage. 
Cuadro 1. Valores medios, desviación estándar y coeficiente variación de la concentración de pigmentos antioxidantes durante 10 días de almacenamiento a diferentes temperaturas y medios.

Table 1. Mean values, standard deviation and variation coefficient of antioxidant pigments concentration during 10 days of storage at different temperatures and preservatives.

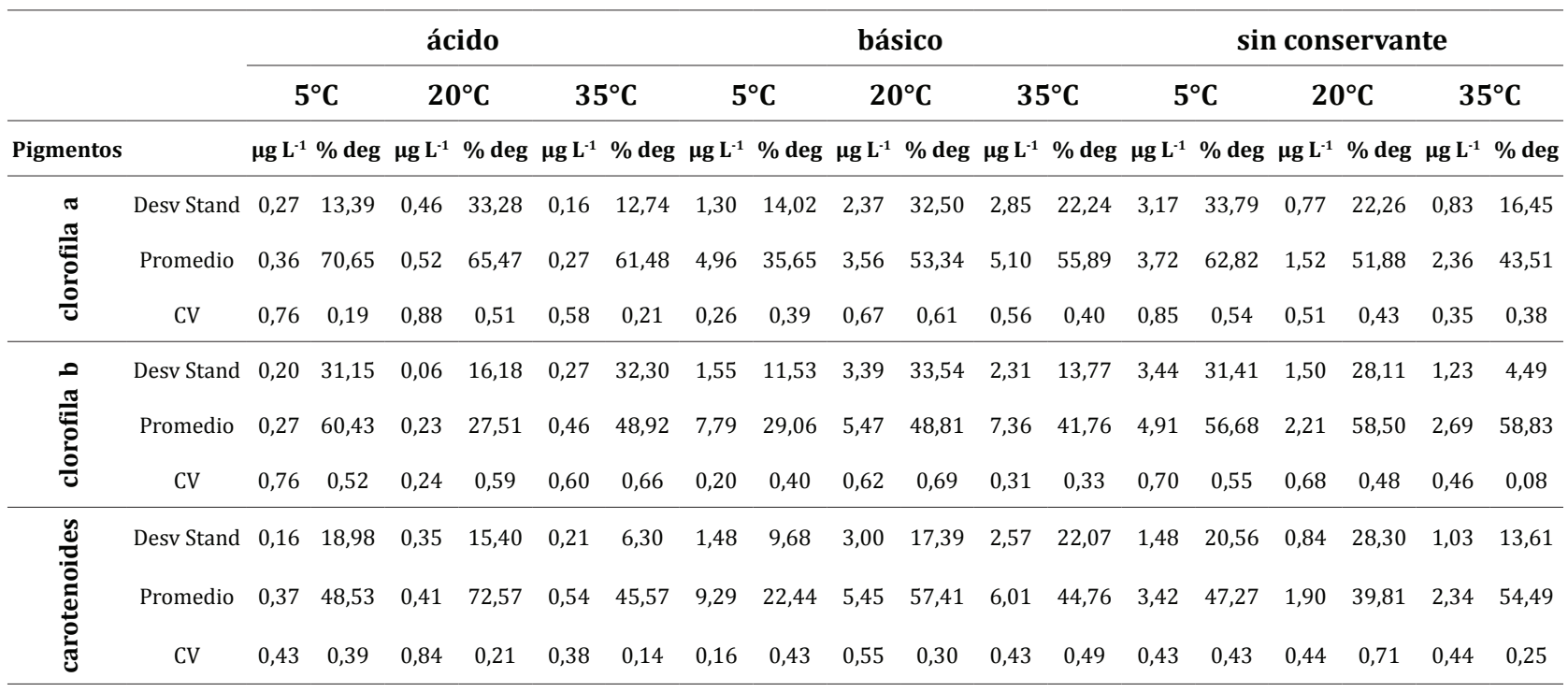

\section{Carotenoides}

Las dinámicas de estabilidad de los carotenoides en los diferentes medios de conservación se asociaron significativamente a regresiones logarítmicas para cada una de las temperaturas de almacenamiento y pueden ser vistas en la Figura 1 (G, H e I). Los carotenoides se degradaron en promedio un $69 \%$ al primer día de almacenamiento, observándose un contenidos de 3,5 a $5,9 \mu \mathrm{g} \mathrm{L}^{-1}$ para 35 y $5{ }^{\circ} \mathrm{C}$. Para el día 10 , el contenido promedio de los medio de $\mathrm{pH}$ mostraron valores de 3,4; 2,6 y 3,0 para 5,20 y $35^{\circ} \mathrm{C}$, respectivamente, lo que en promedio correspondió a una degradación media del $88,8 \%$ para las tres temperaturas utilizadas (Cuadro 1). La temperatura, al igual que el caso de las clorofilas, aunque es un factor significante, participó sólo del $4,6 \%$ de la variación del contenido de carotenoides.

En general, los resultados mostrados sobre la estabilidad de los pigmentos, dan cuenta de una disminución de su valor nutritivo en el zumo de lechuga. Se observó que la mayor magnitud de variación en el contenido de pigmentos activos se debió al efecto inmediato y posterior proceso con degradaciones medias de 71,$6 ; 46,8$ y $69,0 \%$ al primer día de almacenamiento, concordando este efecto con lo descrito en procesos similares por Tijskens et al. (2001). Los resultados en detalle pueden ser vistos en el Cuadro 1, donde en muchos casos no se alcanzó a terminar el proceso de los 10 días por degradación casi total de los pigmentos entre los días seis y nueve.

\section{Efecto del conservante sobre pigmentos: estabilidad y contenidos}

El potencial ácido-base $(\mathrm{pH})$ aportado por el ácido ascórbico, el bicarbonato de sodio se mantuvo inalterado durante el ensayo y en el tiempo de almacenamiento como se puede observar en la Figura 2 con $\mathrm{pH}$ medio de 4,0; 8,3 y 6,3 para los conservantes ácido, básico y control (sin conservante), respectivamente.

En la Figura 2 (A, D y G), se muestra una degradación inmediata y casi total para los tres pigmentos estudiados en soluciones ácidas ( $\mathrm{pH} 4$ ), arrojando una magnitud inicial y final dependientes de la condición del conservante, mostrando para el caso de Chl $a$ valores de 0,85 a $0,10 \mu \mathrm{g} \mathrm{L}^{-1}$, Chl $b 0,54$ a $0,09 \mu \mathrm{g} \mathrm{L}^{-1} \mathrm{y}$ carotenoides 0,81 a $0,25 \mu \mathrm{g} \mathrm{L}^{-1}$. El estudio del análisis de varianza arrojó que el conservante fue responsable del 76,4; 81,5 y 96,2\% de la variación para Chl $a, b$ y carotenoides al primer día de almacenamiento, mientras que para todo el periodo se observó una media de variación de 72,$9 ; 78,8 ;$ y 71,4\% para los mismos pigmentos en el mismo orden. La conversión de clorofilas a feofitinas, se acentúa en condiciones de pH ácido durante el almacenamiento en función del tiempo (Francis, 1964). A su vez, las diferencias en términos de estabilidad entre las clorofilas se debe a un tema de estructuralidad: la sustitución del ión $\mathrm{Mg}^{+2}$ por $\mathrm{Fe}^{+2}$ da lugar a la formación de estos productos como señalan Maiocchi y Avanza (2004) y su degradación aumenta considerablemente por efecto del pH bajo. Estas consideraciones son concordantes con los resultados obtenidos en este ensayo (Schwartz et al., 1999). 
Los pigmentos sometidos a soluciones alcalinas $\mathrm{CpH}$ $8,3)$ mantuvieron una estabilidad superior en y entre ellos durante su almacenamiento (Figura 2: B, E y H), observándose magnitudes iniciales y finales para $\mathrm{Chl} a$ de 7,70 a 2,16 $\mu \mathrm{g} \mathrm{L}^{-1}$; Chl $b 10,00$ a $4,02 \mu \mathrm{g} \mathrm{L}^{-1}$ y carotenoides 10,70 a $3,39 \mu \mathrm{g} \mathrm{L}^{-1}$, lo cual sugiere un efecto de inhibición sobre las clorofilasas evitando así el pardeamiento enzimático acelerado observado en la condición ácida. Gunawan y Barringer (2000) mencionan que incorporar agentes alcalinizantes, tales como bicarbonato de sodio puede ser positivo para la conservación del producto y de los colores brillantes de los pigmentos, lo que es confirmado indirectamente en este trabajo, debido a que se entiende que a mayor contenido de clorofilas, el color verde es más intenso. Esta es una de las razones para elevar el $\mathrm{pH}$ de las hortalizas verdes en medios conservantes, coincidiendo estos resultados con otras investigaciones en otras especies (Gross, 1991; Heaton y Marangoni, 1996; Koca et al., 2007).

Respecto al jugo sin conservante (Figura 2: C, F, I), con ( $\mathrm{pH} \sim 6$ ) de manera general, se observa que en pigmentos $a$ y $b$ aumentan su velocidad de degradación en condiciones de no refrigeración arrojando valores iniciales y finales de 4,95 a 1,05; 4,36 a 1,92 y 4,36 a $1,92 \mu \mathrm{g}$ $\mathrm{L}^{-1}$ para las Chl $a, b$ y carotenoides, respectivamente. En general, los datos observados en el almacenamiento sin aditivos muestran valores medios para el periodo de 10 días de 2,5; 3,5 y 2,5 $\mu \mathrm{g} \mathrm{L}{ }^{-1}$ para los mismos pigmentos en el mismo orden, siendo por tanto este medio de control, el resultante intermedio sobre la estabilidad de los

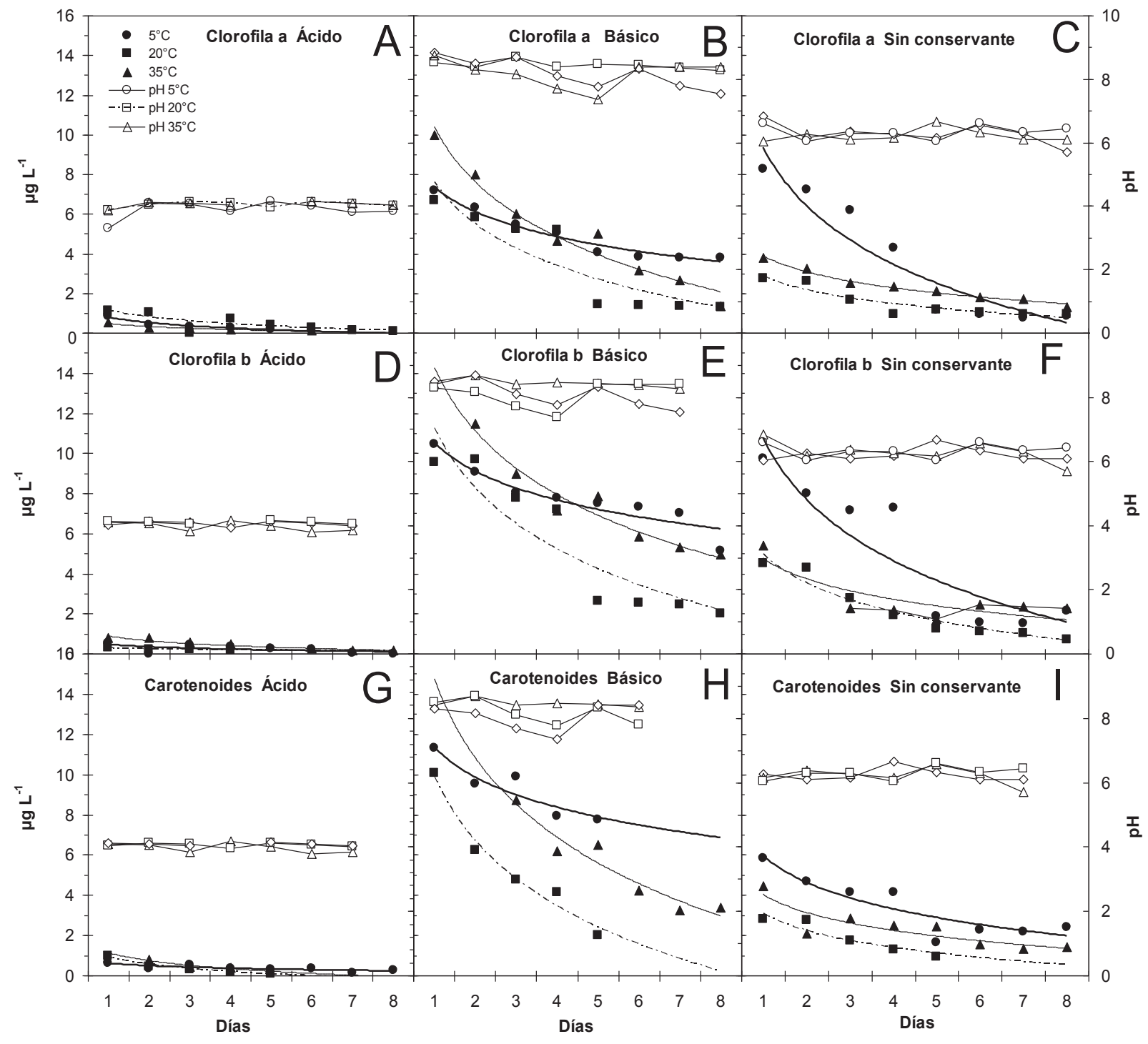

Figura 2. Pigmentos vs. conservante con niveles de la temperatura, en función de los días de almacenamiento.

Figure 2. Pigments vs. conservant with levels of temperature as a function of the days of storage. 
compuestos, precedido por el ambiente básico y seguido por el ácido que siempre arrojó los valores más bajos.

A modo general se puede observar que la degradación mayor se produce al momento de procesar el material, y esto se debe probablemente a la incorporación de oxígeno al momento de la molienda y extracción del zumo o licuado con la consecuente oxidación y desestructuración de las moléculas (Chen y Chen, 1993), lo que da indicios sobre mejoras en el protocolo para la obtención de los pigmentos.

\section{Interacción de los factores y CV}

Las interacciones de ambos factores también resultaron siempre significativas y fuertemente influenciada por el factor de conservación. De este modo, para el día uno, la interacción representó una variación media para todos los pigmentos y temperaturas, del 10,4\%, mientras que para el día 4 y 10, los valores medios fueron de 10,3 y 29,3\% respectivamente, observándose un promedio para todos los días de 18,5\%.

El coeficiente de variación medio para la Chl $a$ en ambientes ácido, básico, alcalino y sin conservante, demostró la movilidad en los datos con valores de 0,74 ; 0,5 y 0,57 , respectivamente, lo que valida la inestabilidad de este pigmeto en condiciones ácidas. Del mismo modo, para Chl $b$, los valores se mostraron menos variantes $(0,53 ; 0,38$ y 0,61 para ácido, básico, alcalino y sin conservante, respectivamente), pero con sensibilidad diferenciada por las características propias de la molécula. Finalmente, los carotenoides mostraron valores medios de CV de 0,55; 0,38 y 0,44 para los mismos conservantes. En general, una variabilidad media del CV 0,6; 0,51 y 0,46 dan cuenta de que las Chl $a$ y $b$ son las más sensibles al almacenamiento, mientras que los carotenoides demostraron tener una mejor estabilidad para los 10 días estudiados con un CV medio entre los conservantes de 0,46 .

\section{CONCLUSIONES}

La temperatura aportó mínimante a la estabilidad del contenido final de clorofilas y carotenoides. Los medios de conservación fueron los que más afectaron la dinámica degradativa de los pigmentos. El medio alcalino fue el que mejor conservó las moléculas de interés, y el medio ácido fue el más destructivo para las mismas.

Se pudo observar además una interacción entre los factores estudiados siendo el factor $\mathrm{pH}$ el responsable de casi un 75\% de la variación del ensayo.

La variabilidad o sensibilidad a la degradación se asociaría a las características propias de cada una de la moléculas, siendo la Chl $b$ más resistente que la $a$, y el carotenoide menos variable que las dos anteriores.

Los resultados permiten concluir que estos métodos de extracción y conservación permiten una muy corta vida útil del producto, debiendo éste ser consumido o utilizado en no más allá de tres a cuatro días de procesado para lograr valores útiles de al menos un 50\%.

Otros estudios son necesarios para determinar niveles organolépticos e inocuidad. Se pueden mejorar las técnicas de laboratorio para obtener resultados donde se busque formular alimentos funcionales ricos en clorofila y con pausada degradación.

\section{REFERENCIAS}

Andrés-Bello, A., Barreto-Palacios, V., García-Segovia, P., MirBel, J., Martínez-Monzó, J., 2013. Effect of pH on Color and Texture of Food Products. Food Engineering Reviews 5(3), 158-170. https://doi.org/10.1007/s12393013-9067-2

Association of Official Analytical Chemist (AOAC), 1980. Official Methods of Analysis of the AOAC. $13^{\text {th }}$ William Horwitz Ed. Washington DC, USA.

Arias, R., Lee, T.C., Logendra, L., Janes, H., 2000. Correlation of lycopene measured by HPLC with the $\mathrm{L}^{*}, \mathrm{a}^{*}, \mathrm{~b}^{*}$ color readings of a hydroponic tomato and the relationship of maturity with color and lycopene content. Journal of Agricultural and Food Chemistry 48(5), 1697-1702. https://doi.org/10.1021/jf990974e

Bertram, J., Bortkiewicz, H., 1995. Dietary carotenoids inhibit neoplastic transformation and modulate gene expression in mouse and human cells. American Journal of Clinical Nutrition 62(6), 1327-1336. https://doi. org/10.1093/ajcn/62.6.1327S

Burns, J., Fraser, P., Bramley, P., 2003. Identification and quantification of carotenoids, tocopherols and chlorophylls in commonly consumed fruits and vegetables. Phytochemistry 62(6), 939-947. https://doi.org/10.1016/ S0031-9422(02)00710-0

Canjura, F., Schwartz, S., Nunes, R., 1991. Degradation kinetics of Chlorophylls and Chlorophyllides. Journal of Food Science 56(6), 1639-1643. https://doi. org/10.1111/j.1365-2621.1991.tb08660.x

Carvalho, P., Machado, C., Moretti, C., Fonseca, M., 2006. Hortaliças como alimentos funcionáis. Horticultura Brasileira 24(4), 397-404. http://dx.doi.org/10.1590/ S0102-05362006000400001

Chen, B., Chen, Y., 1993. Stability of chlorophylls and carotenoids in sweet potato leaves during microwave cooking. Journal of Agricultural and Food Chemistry 41(8), 13151320. https://doi.org/10.1021/jf00032a029

Clark, R., Lee, S., 2016. Anticancer properties of capsaicin against human cancer. Anticancer Research 36(3), 837844.

Dewanto, V., Xianzhong, W., Kafui, A., Rui, L., 2002. Thermal processing enhances the nutritional value of tomatoes by increasing total antioxidant activity. Journal Agricultural and Food Chemistry 50, 3010-3014. https://doi. org/10.1021/jf0115589

Engelhard, Y., Gazer, B., Paran, E., 2006. Natural antioxidants from tomato extract reduce blood pressure in patients with grade-1 hypertension: a double-blind, placebo-controlled pilot study. American Heart Journal 151(1), 100. e1-100.e6. https://doi.org/10.1016/j.ahj.2005.05.008 
Fennena, O., Damodaran, S., Parkin, K., Sanz, B., 2010. Química de los alimentos. Editorial Acribia, S.A., Zaragoza, España.

Francis, F., 1964. pH effect on the high temperature short time processing spinach. Food Technology 18, 1645-1658.

Food and Agriculture Organization of the United Nations (FAO), 1993. Procesamiento de frutas y hortalizas mediante métodos artesanales y de pequeña escala. Oficina regional de la FAO para América Latina y el Caribe. Santiago, Chile.

Greve, L., McArdle, R., Gohlke, J., Labavitch, J., 1994. Impact of heating on carrot firmness: changes in cell wall components. Journal of Agricultural and Food Chemistry 42(12), 2900-2906. https://doi.org/10.1021/ jf00048a048

Gross, J., 1991. Pigments in vegetables: clorophylls and carotenoids. Springer Science \& Business Media, LLC. New York, USA.

Gunawan, M., Barringer, S., 2000. Green color degradation of blanched broccoli (Brassica oleracea) due to acid and microbial growth. Journal of Food Processing Preservation 24(3), 253-263. https://doi. org/10.1111/j.1745-4549.2000.tb00417.x

Hall, M., Jobling, J., Rogers, G., 2013. Influence of storage temperature on the seasonal shelf life of perennial wall rocket and annual garden rocket. International Journal of Vegetable Science 19, 83-95. https://doi.org/10.1080/ 19315260.2012.716387

Halliwell, B., Murcia, M., Chirico, S., Auroma, O., 1995. Free radicals and antioxidants in food and in vivo: what they do and how they work. Critical Review in Food Science and Nutrition 35(1-2), 7-20. https://doi. org/10.1080/10408399509527682

Hayakawa, K., Timbers, G., 1977. Influence of heat treatment on the quality of vegetables: changes in visual green color. Journal of Food Science 42(3), 778-781. https://doi. org/10.1111/j.1365-2621.1977.tb12601.x

Heaton, J., Marangoni, A., 1996. Chlorophyll degradation in processed foods and senescent plant tissues. Review. Trends in Food Science \& Technology 71(7), 8-15. https://doi.org/10.1016/0924-2244(96)81352-5

Hernández, M., Gallego, S., 1999. Tratado de nutrición. Ediciones Díaz de Santos. Madrid, España.

Hormazabal, P., 1999. Efecto de la IV gama en la mezcla de lechuga (Lactuca sativa) tipo escarola y palta (Persea americana Mill) cvs. Edranol, Hass y Negra de la cruz. Taller de Licenciatura, Universidad Católica de Valparaíso, Quillota, Chile.

Izaki, Y., Yoshida, K., Hidaka, K., Toda, K., 1986. Chlorophylls, carotenes and tocopherols in green vegetables and their relationships. Japan Society of Nutrition and Food Science 39(6), 485-493. https://doi.org/10.4327/ jsnfs.39.485

Khoo, H., Prasad, K., Kong, K., Jiang, Y., Ismail, A., 2011. Carotenoids and their isomers: color pigments in fruits and vegetables. Molecules 16(2), 1710-1738. https://doi. org/10.3390/molecules16021710

Koca, N., Karadeniz, F., Burdurlu, H., 2007. Effect of pH on chlorophyll degradation and colour loss in blanched green peas. Food Chemistry 100(2), 609-615. https:// doi.org/10.1016/j.foodchem.2005.09.079

Kufner, R.B., 1980. The biological degradation of chlorophyll in senescent tissues, in: Czygan, F.C., Barz, W. (Eds.), Pigments in Plants, Stuttgart, Germany, pp. 308-313.

Lichtenthaler, H., 1987. Chlorophylls and carotenoids: pigments of photosynthetic biomembranes. Methods in Enzymology 148, 350-382. https://doi. org/10.1016/0076-6879(87)48036-1

Liu, R.H., 2004. Potential synergy of phytochemicals in cancer prevention: mechanism of action. The Journal of Nutrition 134(12), 3479-3485. https://doi.org/10.1093/ jn/134.12.3479S

Liu, Y., Perera, C., Suresh, V., 2007. Comparison of three chosen vegetables with others from South East Asia for their lutein and zeaxanthin content. Food Chemistry 101(4), 1533-1539. https://doi.org/10.1016/j.foodchem.2006.04.005

Maiocchi, M., Avanza, J., 2004. Degradación de clorofilas y feofitinas a diferentes temperaturas en Ilex dumosa e Ilex paraguariensis. Comunicaciones Científicas y Tecnologías. Universidad Nacional del Nordeste, Argentina, Resumen: E-085. http://www.unne.edu.ar/unnevieja/ Web/cyt/com2004/8-Exactas/E-085.pdf

Maunders, M., Brown, S., 1983. The effect of light on chlorophyll loss in senescing leaves of sycamore (Acer pseudoplatanus L.). Planta 158(4), 309-311. https://doi. org/10.1007/BF00397332

Meléndez-Martínez, A., Vicario, I., Heredia, F., 2007. Pigmentos carotenoides: consideraciones estructurales y fisicoquímicas. Archivos Latinoamericanos de Nutrición 57(2), 109-117.

Ministerio de Salud de Chile (MINSAL), 1996. Reglamento Sanitario de los Alimentos. http://www.minsal.cl/ sites/default/files/files/DECRETO_977_96\%20actualizado\%20a\%20Enero\%202015(1).pdf. (acceso, 05.09.2017).

Ministerio de Salud de Chile (MINSAL), 2016. XV Conferencia Iberoamericana de Ministros y Ministras de Salud. http://web.minsal.cl/organizacion-panamericanade-la-salud-la-malnutricion-seria-principal-factor-deriesgo-en-ninos-y-ninas-entre-10-y-14-anos/. (acceso, 05.09.2017).

Pennington, F., Strain, H., Svec, W., Katz, J., 1964. Preparation and properties of pyrochlorophyll a, methyl pyrochlorophyllide a, pyropheophytin a and methyl pyropheophorbide a derived from chlorophyll by decarbomethoxylation. Journal of the American Chemical Society 86(7), 1418-1426. https://doi.org/10.1021/ja01061a030

Prior, R., Cao, G., 2000. Antioxidant phytochemicals in fruits and vegetables: diet and health implications. HortScience 35(4), 588-592.

Sangeetha, R., Baskaran, V., 2010. Carotenoids composition and retinol equivalent in plants of nutritional and medicinal importance: efficacy of $\beta$-carotene from Chenopodium album in retinol-deficient rats. Food Chemistry 119(4), 1584-1590. https://doi.org/10.1016/j.foodchem.2009.09.047

Santos, J., Mendiola, J., Oliveira, M., Ibáñez, E., Herrero, M., 2012. Sequential determination of fat- and water-soluble vitamins in green leafy vegetables during storage. Journal of Chromatography A 1261, 179-188. https:// doi.org/10.1016/j.chroma.2012.04.067

Schoefs, B., 2002. Chlorophyll and carotenoid analysis in food 
products: properties of pigments and methods of analysis. Trends in Food Science \& Technology 13(11), 361371. https://doi.org/10.1016/S0924-2244(02)00182-6

Schwartz, S., Von Elbe, J., 1983. Kinetics of chlorophyll degradation to pyropheophytin in vegetables. Journal of Food Science 48(4), 1303-1306. https://doi. org/10.1111/j.1365-2621.1983.tb09216.x

Schwartz, M., Nuñez, H., Muñoz, A., 1999. Efecto de la temperatura de la pulpa de Kiwi sobre el color, clorofilas y acido ascórbico. Archivos Latinoamericanos de Nutricion. 49(1), 44-48.

Shi, J., Le Maguer, M., 2000. Lycopene in tomatoes: chemical and physical properties affected by food processing. Critical Reviews in Food Science and Nutrition 40(1), 1-42. https://doi.org/10.1080/10408690091189275

Tijskens, L., Barringer, S., Biekman, E., 2001. Modelling the effect of $\mathrm{pH}$ on the colour degradation of blanched broccoli. Innovative Food Science \& Emerging Technologies 2(4), 315-322. https://doi.org/10.1016/S14668564(01)00048-0

Updike, A., Schwartz, S., 2003. Thermal processing of vegetables increases cis isomers of lutein and zeaxanthin. Journal of Agricultural and Food Chemistry 51(21), 6184-6190. https://doi.org/10.1021/jf030350f

Winograd, M., 2006. Intervenciones dentro del programa "5 al día" para promover el consumo de verduras y frutas en Argentina. Revista Chilena de Nutrición 33(1), 282-287. http://dx.doi.org/10.4067/S071775182006000300011.

Xue, L., Yang, L., 2009. Deriving leaf chlorophyll content of green-leafy vegetables from hyperspectral reflectance. ISPRS Journal of Photogrammetry and Remote Sensing 64(1), 97-106. https://doi.org/10.1016/j.isprsjprs.2008.06.002 